\title{
« Jérusalem n'est pas Londres » : les sionistes au lendemain de la Déclaration Balfour dans la presse hébraïque en Palestine
}

\section{Gideon Kouts}

\section{OpenEdition}

1 Journals

Édition électronique

URL : https://journals.openedition.org/tsafon/401

DOI : $10.4000 /$ tsafon.401

ISSN : 2609-6420

Éditeur

Association Jean-Marie Delmaire

\section{Édition imprimée}

Date de publication : 1 décembre 2017

Pagination : 85-94

ISSN : $1149-6630$

\section{Référence électronique}

Gideon Kouts, « « Jérusalem n'est pas Londres » : les sionistes au lendemain de la Déclaration Balfour dans la presse hébraïque en Palestine », Tsafon [En ligne], 74 | 2017, mis en ligne le 31 mai 2018, consulté le 29 juin 2021. URL : http://journals.openedition.org/tsafon/401 ; DOI : https://doi.org/ $10.4000 /$ tsafon.401 


\section{« Jérusalem n'est pas Londres » : les sionistes au lendemain de la Déclaration Balfour dans la presse hébraïque en Palestine}

\section{Gideon Kouts*}

Au lendemain de la Déclaration Balfour, la presse juive et hébraïque en Palestine n'existait quasiment pas. Obligée d'arrêter sa parution en raison de la Grande Guerre, elle a pris du temps pour ressusciter après la conquête britannique. L'armée du général Allenby est entrée dans Jérusalem plus d'un mois après la Déclaration Balfour, le 11 décembre 1917. Mais la censure militaire britannique n'a pas permis la réapparition des journaux avant le 4 avril 1918 ; et encore il s'agissait d'un journal édité au Caire par l'armée : Hadeshot Haaretz. Le premier numéro de Haaretz en tant que quotidien sioniste indépendant est paru à Jérusalem le 18 juin 1919. Le bihebdomadaire du mouvement sioniste travailliste Hapoel Hatzaïr a pu reparaître à Jaffa dès le 22 novembre 1918. Le principal éditeur et rédacteur de la presse hébraïque d'avantguerre, le grand rénovateur de la langue hébraïque Éliezer Ben Yehouda, ainsi que son fils et héritier Itamar Ben-Avi ont rejoint d'abord les rangs de Haaretz, mais non contents de sa ligne trop modérée et hésitante, à leur goût, et sa représentation exagérée de l'alya russe, aux dépens des sionistes déjà installés dans le pays, s'en sont séparés et ont créé leur journal Doar Hayom dont le premier numéro parut le 8 août 1919.

\footnotetext{
* Université Paris 8, correspondant en France de Maariv et Kol Israël. La traduction, à partir de l'hébreu, des extraits et des articles de journaux est de l'auteur.
} 
Face à l'enthousiasme général qui a emporté les cercles juifs sionistes (et non sionistes) dans le monde, ce n'est que sur place, sur les lieux du futur «foyer national», que l'impact immédiat de la grande promesse de la Déclaration Balfour aurait pu être mis à l'épreuve. L'Organisation sioniste a dépêché sur place les 11 membres du «Comité des Députés » (Vaad Hatzirim) d'abord avec Haïm Weizmann lui-même à leur tête, puis remplacé à son retour à Londres par Menahem Ussishkin. Ce comité était censé surtout établir des relations institutionnalisées entre la représentation juive locale et le nouveau pouvoir britannique, mais celui-ci était loin de s'empresser de manifester un désir de collaboration, surtout avec « les sionistes de l'étranger », et les « députés » furent plutôt discrets ; tandis que la direction sioniste centrale à l'étranger a poursuivi les efforts diplomatiques en vue de l'octroi du mandat sur la Palestine à la Grande-Bretagne, les sentiments des sionistes réalisateurs locaux étaient de plus en plus mitigés.

Le 2 novembre 1919, les sionistes du monde entier célèbrent les deux ans de la Déclaration Balfour. À Jérusalem aussi a lieu une réunion solennelle.

Doar Hayom relate ainsi l'événement :

5 novembre 1919

Le lundi, 10 Cheshvan à 5 heures de l'après-midi, le Comité de la communauté des Juifs de Jérusalem a invité les personnalités de notre métropole, ses rabbins et ses intellectuels, à la célébration de la troisième année de la Déclaration Balfour qui a eu lieu dans la grande salle de l'École Lemel ${ }^{1}$.

À l'heure désignée, la grande salle de cette école, éclairée par une lumière luxueuse et décorée des portraits du Grand Dirigeant et des grands personnages de notre peuple, était remplie sans laisser aucun espace vide. Toute la Jérusalem hébraïque, ses communautés et ses partis, ses rabbins et ses intellectuels, était présente à la célébration. Parmi les participants se trouvaient notre invité Yaakov Meir, Grand Rabbin de Salonique, ainsi que des invités d'Égypte.

La célébration s'est ouverte par la chanson « Notre Patrie » jouée par l'Orchestre hébraïque, et Monsieur David Yelin, le Président du

\footnotetext{
${ }^{1}$ École pour jeunes garçons juifs, une des plus anciennes de Jérusalem, fondée en 1856. Jean-Marie Delmaire, De Hibbat-Zion au sionisme politique, Lille, Atelier National de Reproduction des Thèses, 1986, p. 527.
} 
Comité de la communauté, est monté sur scène et a prononcé dans son style riche et brillant et sa belle prononciation orientale le beau discours suivant $[\ldots]$

S'ensuivent des citations du discours, dont voici un passage :

[...] Nous avons tous en mémoire le grand enthousiasme de l'année dernière, et voilà que maintenant nous assistons à la montée de l'affaiblissement de l'esprit et de la dépression, alors qu'il n'y a aucune raison pour cela. En effet, une année a passé sans changements notoires, mais cela du fait de la situation qui n'est pas encore claire concernant d'autres pays... Une année d'observation nous a appris que pour obtenir quelque chose, il faut un travail constant et des efforts incessants. Et celui qui se montre digne de ce qu'on lui donne obtient ce qu'il a besoin d'obtenir. La déclaration britannique est un chèque dont tout Anglais digne de ce nom se voit obligé de calculer la part qu'il va payer. C'est ce que nous avons entendu auprès des cercles gouvernementaux ici et à l'étranger.

Nos dirigeants nous parlent avec assurance et nous leur faisons confiance. Nous devons désormais nous appuyer sur notre travail et nous efforcer de toute notre vigueur. Nous avons le droit de vivre l'époque du début de la rédemption. Nous espérons arriver aussi à mi-chemin et peutêtre aussi à sa fin. Mais les droits impliquent aussi des devoirs. Et chacun de nous doit penser tous les jours : qu'ai-je fait aujourd'hui pour mon peuple, et comment me suis-je rendu utile pour son existence et sa délivrance?

Mais la position qui a prévalu chez des sionistes réalisateurs peut se lire dans un commentaire écrit par Éliezer Ben Yehouda et paru dans le même journal Doar Hayom le 7 novembre 1919, deux jours après le compte rendu de la célébration. Il y relate les cérémonies organisées à Jérusalem et à Londres pour célébrer l'anniversaire de la déclaration et il les compare : l'une modeste à Jérusalem et l'autre fastueuse à Londres. Il en tire des conclusions sur les possibilités de réaliser la Déclaration Balfour à Jérusalem ou depuis Londres et au passage n'hésite pas de revoir à la baisse les superlatifs du compte rendu solennel de l'événement de Jérusalem. 
Doar Hayom : 7 novembre 1919

À Jérusalem et à Londres

Dans les deux capitales les plus grandes et les plus importantes pour nous, à Jérusalem et à Londres, on a célébré l'anniversaire de la Déclaration Balfour.

À Jérusalem, on a célébré - j'emploie ce terme comme une métaphore - cet anniversaire, qui est le jour le plus important dans la nouvelle histoire de notre peuple, comme vous le savez, modestement, presque secrètement, dans une petite salle où se sont rassemblés environ deux cents à trois cents hommes et femmes; l'Orchestre hébraïque joua sur un ton humble une de nos mélodies, deux discours furent prononcés, et c'est par le simple chant de la Hatiqwah ${ }^{2}$ que l'assemblée s'en est retournée chez elle. Il n'y eut personne à cette occasion ni du gouvernement officiel ni même de notre propre gouvernement ${ }^{3}$. Le gouvernement officiel n'envoya même pas aux participants à la cérémonie un mot de félicitations et nous avons reçu de notre gouvernement une petite lettre emplie de sentiments de pleine confiance mais formulée d'un ton réservé.

Telle fut, à Jérusalem, la célébration de l'anniversaire de la Déclaration Balfour qui entrait dans sa troisième année.

Et là-bas dans la capitale du monde, dans la métropole de l'immense Empire britannique on a aussi célébré cet anniversaire, comme vous le savez, comme cela a été publié dans notre presse hier.

Avec éclat et fracas, c'est dans la grande salle luxueuse de l'Opéra de Londres, devant une immense foule de milliers de personnes, parmi lesquelles les plus distinguées de la haute société juive, et aussi non juive, que fut organisée une véritable célébration magnifique de cet important événement. Lord Rothschild ${ }^{4}$ présidait et tous les grands personnages du monde juif ainsi que tous les leaders sionistes étaient présents à cette occasion; des hommages et des excuses sont parvenus de la part des représentants du gouvernement officiel, du nouveau ministre des Affaires étrangères Curzon ${ }^{5}$, de Sir Lord Robert Cecil ${ }^{6}$ et du ministre de l'Outre-

\footnotetext{
${ }^{2}$ L'espérance, chant sioniste écrit en 1878 par Naftali Hertz Imber, la musique, adaptée d'une mélodie populaire de Roumanie, est de Samuel Cohen. Il exprime l'espoir, vieux de deux mille ans, du peuple juif de pouvoir vivre à nouveau sur la Terre d'Israël, Eretz Israël. C'est actuellement l'hymne national israélien.

${ }^{3}$ Le « Comité des Députés ».

${ }^{4}$ Lord Lionel Walter Rothschild (1868-1937) à qui fut adressée la lettre de Balfour présentant sa déclaration.

${ }^{5}$ George Nathaniel Curzon (1859-1925), homme politique anglais appartenant au parti conservateur. Il fut vice-roi des Indes et secrétaire aux Affaires étrangères de 1919 à 1924. Ce fut lui qui négocia la frontière entre la Pologne et la toute nouvelle URSS, en 1920, laissant son nom à la ligne qui sépara les deux pays.
} 
mer, qui ne pouvaient pas être présents à cette célébration. Et tous, presque unanimement, ont dit clairement que les sentiments du gouvernement d'Angleterre n'ont pas changé non plus que ses intentions en ce qui concerne la création d'un foyer national pour le peuple d'Israël sur la terre de ses ancêtres, et que c'est uniquement la situation compliquée des intérêts de politique générale qui a causé un retard dans la mise en place officielle de la Déclaration Balfour ${ }^{7}$; et pourtant dès que la paix fut signée avec la Turquie ${ }^{8}$ et que la responsabilité sur la Palestine fut donnée de manière officielle à l'Angleterre, le gouvernement commença immédiatement à œuvrer à l'application de la Déclaration Balfour et à aider en cela les Juifs, de tout son pouvoir.

Une telle célébration dans la métropole de l'Angleterre, au moment des événements d'Égypte, au milieu des cris et des menaces de la part du «Cadi» de Damas ${ }^{9}$, les paroles claires des représentants officiels du gouvernement anglais durant cette célébration suffisent à calmer les esprits les plus incrédules parmi nous.

Cette différence dans la manière de célébrer cet anniversaire à Jérusalem et à Londres est la preuve évidente qu'il y a une différence d'opinions entre les cercles officiels à Jérusalem et à Londres. Il n'y a aucun doute que nous préférons tous cette différence à son contraire. Pourquoi cette différence ? Ce n'est pas le lieu ici d'approfondir ce sujet. Bien sûr dans une certaine mesure cette différence est liée à la différence des lieux, à l'état d'esprit d'une partie des habitants ici auquel tout le monde admet qu'il faut prêter attention. Mais il ne faut pas dissimuler le fait que d'autres raisons font que le gouvernement officiel local traite prudemment la Déclaration Balfour, peut être au-delà des limites convenues. Pour le public juif ici, cette extrême prudence de la part du gouvernement local occasionne une grande détresse morale, et il n'y a rien d'étonnant si cet état d'esprit frôle parfois le désespoir. Mais chaque

\footnotetext{
6 Lord Robert Cecil (1864-1958) était, en 1919, secrétaire d’État aux Affaires étrangères. Homme politique britannique, avocat et diplomate, il fut un des artisans de la Société des Nations, ce qui lui valut le prix Nobel de la Paix en 1937. Ses excuses, ainsi que celles de Curzon, sont la marque de l'importance accordée à cette cérémonie à Londres par contraste avec ce qui s'est passé à Jérusalem.

${ }^{7}$ Allusion à la réticence de la part de l'Angleterre à diffuser la déclaration en Palestine qui venait juste de sortir de l'Empire ottoman.

${ }^{8}$ La Turquie avait pris part à la Première Guerre mondiale aux côtés de l'Allemagne et c'est l'Angleterre qui participa à sa défaite avec la conquête de la Palestine par le général Allenby. C'est donc avec la Turquie que l'Angleterre doit négocier la paix en Palestine.

9 Durant l'année 1919, les Égyptiens qui ont contribué à l'effort de guerre de l'Angleterre contre la Turquie manifestent par des grèves et dans les rues pour obtenir leur indépendance. Dans le même temps, la population de Damas s'agite pour les mêmes raisons.
} 
information provenant de là-bas, de Londres, annonce et montre clairement que là-bas telle n'est pas la situation, car notre destin est làbas, entre les mains du gouvernement suprême qui lui décide, et lui seul ; là-bas à Londres le gouvernement et le peuple voient dans la Déclaration Balfour un des fondements de la politique anglaise, et s'y exprime une ferme décision pour mettre en pratique cette déclaration.

Et cette différence entre Jérusalem et Londres, nous ne devons pas l'oublier même une seule seconde. Nous ne sommes pas spécialistes du comportement du gouvernement anglais, et il nous est difficile de comprendre comment dans un gouvernement européen il peut y avoir une telle différence d'attitude entre le gouvernement central et ses représentants d'Outre-mer. Nous sommes habitués à l'ordre de gouvernements centralisés jusqu'au dernier degré, des gouvernements dont tous les rouages de la mécanique mettent en branle, par la force du mouvement central unique, un mouvement qui se transmet à tous les autres rouages d'un seul coup. Mais il semble que le gouvernement d'Angleterre n'est pas centralisé à ce point. Est-ce un défaut ou une qualité ? L'histoire de cette nation témoigne au moins d'une chose, en procédant de la sorte, elle est parvenue à étendre son pouvoir sur d'immenses pays lointains et elle sait les conserver en son pouvoir. Et cette qualité du gouvernement anglais nous devons nous en souvenir en toute occasion, en tout événement qui nous émeut plus ou moins justement. Il n'y a pas de doute que cette différence va se réduire au fur et à mesure que la Déclaration Balfour commencera à se concrétiser. Le gouvernement local qui est actuellement entièrement militaire évoluera en gouvernement civil et son rôle officiel sera de nous aider dans la réalisation de la déclaration. Ce futur gouvernement sera, probablement, plus proche en pensée du gouvernement central, et saura bien pourquoi il a été institué ici et quels sont ses devoirs envers nous. Mais là aussi il est possible qu'il y ait encore quelque différence entre Jérusalem et Londres. Peut-être tous les fonctionnaires ne connaîtront pas ou ne voudront pas connaître les buts et les intentions du gouvernement central. Des faits peu agréables et plus ou moins importants également pourraient se produire de temps en temps. Quoi qu'il en soit nous devons nous souvenir d'une chose : Jérusalem n'est pas Londres.

C'est une règle très importante pour nous maintenant, au seuil de notre vie nouvelle. Dans peu de temps commencera cette vie nouvelle. Dans quelques mois résonnera le grand shofar qui proclamera partout dans le monde à tous les habitants de ce pays qu'Eretz Israël est véritablement la terre dont doit hériter Israël ${ }^{10}$. Mais le monde poursuit

\footnotetext{
${ }^{10}$ Comparaison avec la sonnerie du shofar qui, dans la religion juive, annonce le nouvel an et donc un recommencement dans la vie de toute personne juive, et de la même
} 
son chemin. Et cette révolution si grande et immense ne se fera pas facilement, soudainement, en une seule fois, sans obstacles, comme par enchantement. Sur ce chemin difficile, très difficile, nous devons progresser lentement, nous devrons avoir en tête chaque jour ce point essentiel : toutes les actions du gouvernement local ne s'accorderont pas avec les objectifs et les intentions du gouvernement central.

Ici, à Jérusalem, nous avons célébré modestement l'anniversaire de la Déclaration Balfour, et c'est avec honneur et splendeur qu'il a été fêté, avec la participation des représentants du gouvernement central, là-bas à Londres. Ce qui vient de se passer doit être, pour nous, dès maintenant, un signal et un exemple dans notre nouvelle vie. Les paroles que les représentants du gouvernement anglais nous ont adressées lors de cette célébration nous apaisent, sûrement, pour tout ce qui peut nous arriver ici. Ce n'est pas de ceux-là que viendra notre vie nouvelle. De telles paroles explicites ont été prononcées de manière tellement officielle et à une heure si grave qu'elles ne laissent aucune place pour le doute et l'incrédulité. Les ministres du gouvernement de la Grande Bretagne ne parlent pas de la sorte pour se moquer du peuple entier. La voix que nous entendons de là-bas, de Londres, est tellement importante, forte et merveilleuse, que d'un esprit tranquille et d'un cœur assuré nous pouvons regarder ce qui se passe ici, même si tout ne se déroule pas comme nous le souhaiterions.

Choisir la discrétion, attendre l'aboutissement du processus diplomatique et entre-temps ne pas arrêter d'agir sur le terrain - ne pas se laisser impressionner par Jérusalem et attendre les bonnes nouvelles de Londres - telle était la ligne de conduite recommandée par le noyau dur des sionistes de tout bord, dont Ben Yehouda se faisait écho.

$\mathrm{Au}$ bout d'un an, l'évolution de l'actualité " sioniste » au niveau international, la conférence de San Remo qui a conditionné l'octroi du mandat sur la Palestine par la Grande-Bretagne par la mise en œuvre de la Déclaration Balfour, mais aussi en Palestine, avec la nomination de Sir Herbert Samuel en tant que premier Haut-Commissaire de la Palestine, semblent lui avoir donné raison. Le 2 novembre 1920, il écrit un éditorial beaucoup plus enthousiaste et optimiste, dont voici quelques extraits. La différence entre 1919 et 1920 réside à ses yeux dans ces deux faits majeurs :

manière ce grand shofar annoncera la nouvelle vie du peuple d'Israël héritant d'un foyer national. 
Doar Hayom : 2 novembre 1920

Quatrième année de la Déclaration Balfour

Aujourd'hui c'est le $1^{\text {er }}$ jour de la quatrième année de la Déclaration Balfour. Mais ce Jour de l'An ne ressemble pas à ses trois frères qui l'ont précédé. Ce Jour de l'An est le premier après San Remo, le premier sous le Commissariat de Herbert Samuel ${ }^{11}$. Et ces deux événements donnent à ce Jour de l'An de la Déclaration Balfour, sa forme, sa beauté...

En ce grand jour heureux, quand la lettre de Balfour a été publiée [...] il y avait plusieurs personnes non seulement parmi les non-juifs, mais aussi, et peut-être surtout, en notre sein, qui ont méprisé cet acte, qui lui ont ôté toute valeur historique; qui n'ont vu en lui qu'un ramassis de ruses de guerre de la part de l'Angleterre, la rusée. Et je peux témoigner que même les plus amicaux envers l'Angleterre parmi nous, ceux qui la désirent le plus, qui lui accordent le plus leur confiance, même eux ont eu des doutes cachés. Ces mots peu nombreux, simples, comme s'ils traitaient d'une affaire légère et quotidienne, ont parlé d'une manière si magnifique et si extraordinaire dans le comportement convenu des nations, et nous particulièrement, qu'en effet il était difficile d'y voir un événement de grande valeur [...] Les contestations récentes du texte du Mandat témoignent elles aussi de la grandeur de la déclaration, parce que les contestataires eux-mêmes ont reconnu clairement que dans le document du Mandat il y a quelques modifications du texte de la déclaration.

Et c'est cela qui fait la grandeur de cet anniversaire de la Déclaration de Balfour. C'est son premier anniversaire après avoir déjà rempli son premier rôle à San Remo et après un certain temps d'expérience pratique et l'éclat de sa splendeur non seulement n'a pas été abimé mais il brille maintes fois plus, et il n'y a aucun doute que d'une année à l'autre, jusqu'à la fin des siècles sa splendeur ira en grandissant $[\ldots]$

Mais le pouvoir mandataire continua de s'éloigner de la déclaration originale, qui devint avec le temps la référence d'une vague promesse

\footnotetext{
${ }^{11}$ Lord Herbert Samuel (1870-1963), politique anglais, ministre de l'Intérieur durant une courte durée de la Première Guerre mondiale. Juif, il fut proche des sionistes anglais et de Weizmann, il adhéra au Congrès sioniste anglais. De 1920 à 1925 il fut Haut-Commissaire britannique en Palestine, suscitant de grands espoirs dans les rangs sionistes et la population juive de Palestine.
} 
ancienne d'avenir radieux. Le mouvement sioniste ne fut pas non plus satisfait du fonctionnement des Hauts-Commissaires de la Palestine suivants. La période qui s'ouvrit jusqu'à la création de l'État d'Israël fut marquée par un long travail de faits accomplis et d'épreuves de force diplomatiques mais aussi militaires avec les voisins arabes et la puissance mandataire, sans compromettre l'importance historique de la déclaration comme pilier de la « reconnaissance internationale » dans la légitimation du discours sioniste. 
Éliezer Ben Yehouda à sa table de travail (site : kefisrael.com)

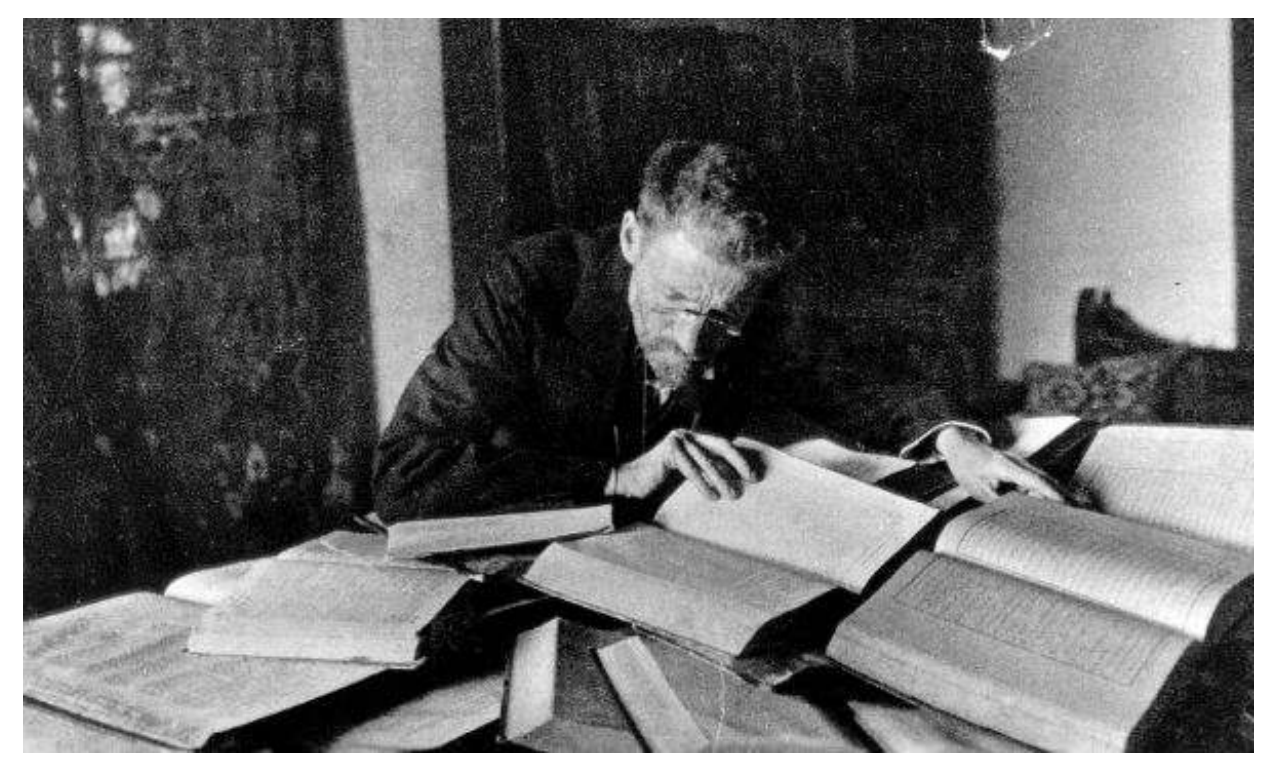

Éliézer Ben Yehouda (Biélorussie 1858 - Jérusalem 1922) était un journaliste et philologue. C'est principalement lui qui œuvra à l'usage quotidien de la langue hébraïque par les pionniers de Palestine. Il est donc à l'origine de l'hébreu parlé moderne, après la renaissance de l'hébreu par le mouvement de la Haskala (XVII $-\mathrm{XIX}^{\mathrm{e}}$ siècles). Son fils Itamar Ben-Avi fut le premier enfant juif de Palestine pour qui l'hébreu fut la langue maternelle. 